Mots. Les langages du politique

\title{
Du parti de la panthère noire aux panthères : un ou des Black Panther Party(ies)?
}

From the Black Panther Party to the Panthers: one or some Black Panther Party(ies)?

Del partido de la pantera negra a las panteras: ¿uno o varios Black Panther Party(ies)?

\section{Valérie Bonnet}

\section{OpenEdition}

\section{Journals}

Édition électronique

URL : https://journals.openedition.org/mots/25303

DOI : $10.4000 /$ mots. 25303

ISSN : 1960-6001

Éditeur

ENS Éditions

\section{Édition imprimée}

Date de publication : 11 juillet 2019

Pagination : 127-143

ISBN : 979-10-362-0170-7

ISSN : 0243-6450

\section{Référence électronique}

Valérie Bonnet, « Du parti de la panthère noire aux panthères : un ou des Black Panther Party(ies) ? », Mots. Les langages du politique [En ligne], 120 | 2019, mis en ligne le 01 janvier 2022, consulté le 22 avril 2022. URL : http://journals.openedition.org/mots/25303; DOI : https://doi.org/10.4000/mots.25303 


\section{Du parti de la panthère noire aux panthères : un ou des Black Panther Party(ies)?}

Le Black Panther Party (1966-1982), fondé par Huey P. Newton et Bobby Seale, deux anciens étudiants du collège universitaire d'Oakland, est une organisation qui marqua durablement le paysage de l'activisme africain-américain. C'est sans doute en raison de la scénographie qu'elle choisit, marquée par une image martiale (tenues paramilitaires, port ostensible d'armes à feu) et un mode d'action légaliste ${ }^{1}$ (patrouilles armées circulant dans les rues des quartiers noirs en vertu du deuxième amendement de la Constitution des États-Unis, rappels à la loi des officiers de police) que cette organisation eut très rapidement les faveurs des caméras. La mise en place des manifestations anti-guerre visant à lutter contre la conscription des Africains-Américains et autres minorités, tout comme un soutien aux jeunes gens essayant d'échapper à celle-ci, lui valurent la sympathie de mouvements étudiants. Elle œuvra également au service de la communauté par l'éducation populaire dans divers domaines, l'accompagnement des familles de détenus, la lutte contre l'alcoolisme, la toxicomanie et la drépanocytose (maladie touchant spécifiquement les populations noires). Mais son opération la plus connue demeure le Free Breakfast for Children Program organisé dans la baie de San Francisco. En effet, le groupe chargé de ce programme, qui reprenait les pratiques d'auto-organisation des populations noires, visait également l'introduction du socialisme dans les ghettos par des actions concrètes.

Ainsi, son manifeste en dix points, le Platform and Program, s’il fut inspiré de celui de la Nation of Islam, n'adopta pas pour autant le séparatisme de cette dernière, position que l'on peut autant imputer à un marxisme aux soubassements internationalistes (internationalisme également irrigué par la

1. Les modes d'action du Black Panther Party sont empruntés au Deacons for Defense and Justice, groupe d'autodéfense fondé par des vétérans de Jonesboro (Louisiane) en 1964. S'ils ne furent pas les premiers à utiliser les armes, ils furent les premiers à le faire de manière organisée. Les Deacons for Defense and Justice furent une source d'inspiration sur le point de l'autodéfense armée de la Lowndes County Freedom Organization (1965), qui luttait pour la participation électorale des Africains-Américains.

Université Toulouse 3-Paul Sabatier, LERASS

valerie.bonnet@free.fr

Mots. Les langages du politique $\mathrm{n}^{\circ} 120$ juillet $2019 \bullet 127$ 
pensée de Franz Fanon, de Robert F. Williams et de Malcolm X) qu'à un certain pragmatisme (ainsi que l'indique l'alliance avec la Nouvelle Gauche). Il défendait un internationalisme prolétarien où la lutte des classes supplante la lutte des races, et, adossé à son répertoire d'action bien spécifique, contribua à la notoriété du Black Panther Party, y compris hors des frontières états-uniennes.

Ce parti fit en effet florès idéologiquement, mais aussi onomastiquement, puisque son nom se vit décliné et réutilisé dans les dénominations de divers mouvements se reconnaissant dans son discours nationaliste mêlé d’inspirations marxistes-léninistes. Ces variations paradigmatiques soulignent la prégnance du Black Panther Party (désormais BPP) dans le panorama de l'activisme mondial comme elles montrent la charge symbolique dont ce nom est porteur ${ }^{2}$. Les différentes désignations en discours - au fil des textes, cette organisation est désignée par the Black Panther Party, the Black Panthers, the Panthers - mettent quant à elles en évidence les logiques de structuration à l'œuvre au sein du parti comme de la nébuleuse mondiale qu'il initia.

Ce sont donc les variations paradigmatiques et syntagmatiques de ce nom ainsi que les enjeux politiques, discursifs et représentationnels de celles-ci que nous nous proposons d'exposer dans cet article.

On constate en effet qu'en discours, ce n'est pas seulement la désignation, mais également la dénomination du BPP et de ses avatars qui manque de stabilité. L'analyse des paradigmes désignationnels nous permettra de déterminer le rôle discursif des signifiants et les relations sémantiques entre les différentes dénominations. Ces modifications, qui interviennent tant sur le plan syntagmatique (morphologique) que lexico-sémantique (modification des spécifiants/qualifiants) et onomasiologique (modification de l'origo3), sont révélatrices des (auto)représentations de cet objet qu'est le BPP. Leur étude nous permettra non seulement de dégager la signifiance de ce nom propre et les modalités de cette signifiance, elle montrera également que la frontière entre dénomination et désignation est, dans le cas présent, labile4.

\section{Un nom peu commun}

La dénomination du Black Panther Party est le fruit d'une hétéro-attribution à imputer à la presse au service de ses adversaires politiques : celle-ci désignait la Lowndes County Freedom Organization par le symbole qu'elle arborait, une

2. Les Gray Panthers, fondées en 1970 pour la défense des droits des personnes âgées, la Purple Panther Division rebaptisée Lavender Panthers, groupe d'autodéfense armée LGBT (1973-1974), ne sont pas d'obédience marxiste-léniniste. Si certains de leurs moyens d'action ou de leur cause rejoignent ceux du BPP, ils peuvent difficilement être rattachés à la lutte des aborigènes ou des Maoris avec lesquels les Africains-Américains forment le Black Atlantic et le Black Pacific.

3. L'origo est le repère sur lequel s'appuie la compréhension de la deixis.

4. De ce fait, ce travail s'appuie sur un corpus « au vol» (Moirand, 2018). 
panthère noire5. Comme le souligne Stokely Carmichael, cette manière d'agir oscillait entre déni et condescendance :

Now the gentlemen of the Press, because they're advertisers, and because most of them are white, and because they're produced by that white institution, never called the Lowndes County Freedom Organization by its name, but rather they call it the Black Panther Party. Our question is, Why don't they call the Alabama Democratic Party the "White Cock Party"? (It's fair to us...) It is clear to me that that just points out America's problem with sex and color, not our problem, not our problem. (Carmichael, 1966)

Ce surnom ainsi que ce symbole touchèrent l'imagination de H.P. Newton et B. Seale, qui, part par provocation, part par retournement de stigmate, part par réel engouement (ou pragmatisme), demandèrent le droit de les utiliser au Student Nonviolent Coordinating Committee (SNCC), sous l'égide duquel la Lowndes County Freedom Organization avait été fondée. Ils ne furent pas les seuls à avoir cette idée, puisque le Harlem Black Panther Party (1966-1967) et le Black Panther Party of Northern California (connu aussi sous le nom des San Francisco Panthers, 1966-1967 ) naquirent la même année ; pour se différencier de ces derniers, les deux fondateurs y adjoignirent l'extension nominale for Selfdefense. Celle-ci permettait de surcroit de mettre en exergue leur programme d'action, hérité non seulement de l'expérience de terrain, mais aussi des pensées de F. Fanon, de R. F. Williams et de Malcolm X, comme nous l'avons indiqué. Au regard de la faible pérennité de ces homonymes, cette précision disparut rapidement (dès 1967), sous l'influence d'Eldridge Cleaver, qui venait de rejoindre le parti et impulsa la mise en place de la seconde partie du programme du BPP, l'organisation communautaire.

Ce nom n'est donc pas totalement lié à un choix revendiqué; ou plutôt il s'agit d'un choix revendiqué par provocation. Désignation ironique adoptée comme dénomination polémique, elle a pour conséquence de ne pas rendre réellement compte des positions et modes d'action de l'organisation qu'elle étiquète. En effet, le BPP ne visait pas particulièrement les scrutins : en raison de ses assises marxistes-léninistes, son objectif était la révolution, les candidatures de certains de ses membres ayant des visées essentiellement symboliques7. Le terme party indique ici davantage les forts soubassements idéologiques d'une organisation qui aspire à se différencier de ses alter ego africaines-américaines, souvent sous l'égide de congrégations religieuses.

5. Les partis se devaient d'avoir un symbole, à destination des publics illettrés.

6. B. Seale (1991, p.113) affirme lui-même ne pas trop savoir lequel de ces groupes émergea le premier. Quoi qu'il en soit, le Black Panther Party for Selfdefense affichait son mépris envers les membres du Black Panther Party of Northern California en les surnommant les Paper Panthers, en référence aux paper tigers dénoncés par Mao Tse Dong, autre influence idéologique de H.P. Newton.

7. Ainsi en est-il de la candidature de H.P. Newton, alors emprisonné, aux élections au Congrès en 1968. 


\section{Une diffusion onomastique}

Comme signalé plus haut, l'internationalisme du BPP le conduisit à essaimer diversement dans les communautés en quête d'autodétermination et de respect. Si le cadre idéologique de l'organisation est inégalement adopté, son TenPoint Program et son nom se virent adaptés aux problématiques locales. Citons le White Panther Party (1968, collectif antiraciste et anti-impérialiste étatsunien), le British Black Panther Movement (1968, mouvement afro-caribéen), l'Australian Black Panther Party (1969, mouvement aborigène), le Polynesian Panther Party (1971, mouvement maori), les Black Panthers of Israel (1971, mouvement de défense des juifs mizrahim), les White Panthers UK (1971), les Dalit Panthers (1972, mouvement des Harijans), mais aussi le Black Beret Cadre (1969, mouvement de défense des populations noires des Bermudes) ou les Brown Berets (1967, jeunesse chicano de Los Angeles).

\section{Un point de vue paradigmatique}

Les origines et motivations de ces dénominations sont diverses : les hapanterim ashrorim (littéralement "panthères noires») se virent attribuer le surnom de «panthères noires israéliennes» par la presse après qu'ils eurent déclaré vouloir être «comme les Black Panthers pour l'État d'Israël». À l'instar de leurs alter ego états-uniens, ils reprirent ce surnom dont ils supprimèrent plus tard l'extension géographique spécifiante, la presse prenant quant à elle l'habitude de supprimer les guillemets d'ironie. De fait, ce nom, choisi dans l'optique de focaliser l'attention et non par réelle connaissance des options idéologiques du BPP, qui soutenait la cause palestinienne ${ }^{8}$, heurta la sensibilité de certains. L'adoption d'une hétéro-attribution fut également le fait du Young Chicanos for Community Action, mouvement de la jeunesse mexicaine de Los Angeles, qui endossa le surnom de Brown Berets que lui assigna la police, ou du parti maori qui se vit affubler du slogan de Brown Power par la presse grand public.

Le White Panther Party doit son nom à une question posée à H. P. Newton lui demandant si la formation d'un White Panther Party, qui lutterait contre les discriminations subies par les blancs pauvres, était envisageable (Newton, 1968)9. La réponse allusive que fit alors le responsable du BPP fut interprétée comme

8. Ce qui leurvalut de subir diverses pressions et de recevoir quelques propositions alternatives à ce nom controversé, dont les Black Lions of Judea. Signalons qu'en 1973, ils décidèrent de candidater aux élections sous la bannière des Black Panthers-Israeli Democrats, une faction rivale s'établissant comme le «Real Panthers» Party.

9. La légende circulant à propos de cet échange est légèrement différente et révélatrice de la force paradigmatique de la dénomination comme de l'importance d'une attribution onomastique. Elle veut en effet que ce collectif se soit formé à la suite de la réponse « qu'ils fondent un White Panther Party» à la question « que peuvent faire les Blancs pour aider le BPP?». 
une invite, et John Sinclair, le manager du MC5, groupe de la scène d'Ann Arbor (Michigan), constitua le collectif majoritairement estudiantin qu'il nomma le White Panther Party. À la suite de celui-ci-ci se formèrent les White Panthers UK.

Ainsi, les dénominations issues du syntagme black panther party semblent autant relever d'auto- que d'hétéro-attribution. En se saisissant de désignations connotées négativement les mouvements se réapproprient et inversent un stigmate jeté tant par des adversaires politiques que par une presse rarement favorable. Ceux-ci, adoptant une logique de comparaison implicite par l'utilisation de structures antonomasiques ${ }^{10}$, désignent des mouvements en référence à ce qui semble être un parangon. On remarque la prégnance et le maintien du substantif panther, majoritairement en collocation avec black. Cet adjectif de couleur est parfois remplacé par des spécifiants de classe (Dalit) ou d'origine (Polynesian). Seul, le mouvement des Bermudes choisit beret, en hommage, selon Mumia Abu Jamal, à la tenue des activistes états-uniens ${ }^{11}$. La conservation du paradigme adjectival de couleur permet quant à elle de référer au BPP. La forte structuration militaire est montrée par l'holonyme ${ }^{12}$ cadre («petit groupe militaire»), qui forme une isotopie avec beret (coiffure militaire). Globalement, party se voit remplacé (movement, cadre), ou supprimé.

\begin{tabular}{l|l|l|l|l}
\hline & White & Panther & Party & \\
\hline & Black & Panthers & & (of Israël) \\
\hline British & Black & Panther & Movement & \\
\hline & White & Panthers & & Of the UK \\
\hline Australian & Black & Panther & Party & \\
\hline Polynesian & & Panther & Party & \\
\hline & Dalit & Panthers & & (Of India) \\
\hline & Black & Beret & Cadre & \\
\hline & Brown & Berets & & \\
\hline
\end{tabular}

Tableau récapitulatif des noms de groupes se réclamant du BPP

Le tableau ci-dessus permet de percevoir le poids de cette organisation dans les imaginaires de lutte. En effet, la régularité du paradigme constitue un indice de sa diffusion, qui, en retour, renforce la solidité de la synapsie ${ }^{13}$ :

On peut donc constater une interaction entre le modèle producteur et la diffusion du modèle dans le corps social. Il se produit une multiplication des unités syntagmatiques dans le lexique par la différentiation des bases, correspondant à l'expérience sociale, et par la génération à partir de l'élément de détermination. (Guilbert, 1975, p. 261)

10. Ici $[\mathrm{det}+\mathrm{Np}+$ préposition + toponyme] ou [det + adj relationnel + Np]. Exemples : the Jerusalem of the Balkans, the British Leonard Cohen.

11. Et des guérillas tiers-mondistes (Ogbar, 2006).

12. Terme désignant un tout composé de parties elles-mêmes désignées par un méronyme.

13. Locution dont les éléments sont reliés par des rapports de subordination. 
On remarque que Dalit, mis en équivalence avec black, souligne la dimension stigmatisante perçue dans la couleur de peau, et l'inversion de stigmate afférente ${ }^{14}$. Cette affirmation du groupe désigné par l'adjectif catégorisant posa problème au White Panther Party, dont le fondateur, J. Sinclair, dut lutter pour que l'organisation ne soit pas reçue comme un parti suprémaciste blanc ${ }^{15}$. Cet adjectif peut en effet avoir des fonctions différentes : caractérisant comme dans le cas de Polynesian (Panther Party) ou méronyme dans celui de Australian (Black Panther [Party]) qui semble n'être qu'une branche du groupe fondateur, comme le laisserait supposer l'internationalisme de celui-ci. Si l'un précise le lieu d'origine du groupe intitulé Panther Party, l'autre, en reprenant l'intégralité de la dénomination états-unienne, lui confère le statut d'holonyme tout en lui faisant perdre son enracinement géographique ${ }^{16}$.

\section{Collections et classes}

On notera par ailleurs que les dénominations oscillent entre une utilisation au pluriel ou au singulier du lexème panther/beret (British Black Panther Movement vs White Panthers of the UK; Black Beret Cadre vs Brown Berets), ce qui implique une vision différente des organisations qu'ils désignent. Morton E. Winston, Roger Chaffin et Douglas Herrmann (1987, p. 423) proposent de discriminer les noms collectifs entre noms de classes et de collections. Les classes sont déterminées par la similarité de leurs membres alors que les collections le sont par les relations de proximité spatiale ou les relations sociales. En d'autres termes, les collections se constituent sur la base des caractéristiques extrinsèques à leurs membres alors que les classes sont fondées sur des caractéristiques intrinsèques communes (ibid., p.428). Le pluriel favorise une perception en classe, l'extension en complément du nom géographique (of Israel, of India, of the UK) renvoyant au lieu d'origine de celle-ci. L'adjectif relationnel catégorisant Australian, Polynesian, British, plus formel, souligne la structure de party. On constate en effet que le singulier panther accompagne systématiquement un substantif signifiant une forme d'organisation, qu'il détermine (party, movement, ou dans le contexte des Bermudes, cadre). Il semble le relier à la structure d'origine, le BPP californien, et composer ainsi ce qui constituerait une filiale. De fait, le spécifiant adjectival change de statut avec la structuration du syntagme ; il détermine l'organisation dans le premier cas, alors qu’il détermine les membres de celle-ci dans le second:

14. Dans les années 1960, dalit («cassé, brisé») est une autodénomination remplaçant intouchable, terme utilisé par les Brahmanes.

15. Ainsi, un groupe de policiers new-yorkais opposés aux BPP formèrent les White Tigers. On constate l’opposition paradigmatique révélatrice des antagonismes politiques.

16. La possibilité en discours de trouver des formulations comme American Black Panther Party corrobore ce point. 
British (Black Panther [Party]) vs (Black [Panthers]) of Israel

À un accent mis sur la structure (party) s'oppose un accent mis sur l'essence (couleur de peau ou caste) ${ }^{17}$. Ce choix semble lié à la représentation que se font les groupuscules de leur structuration nationale et internationale : les Black Panthers of Israel, les Brown Berets, les Dalit Panthers of India ${ }^{18}$ référent à l'action de l'organisation états-unienne par le truchement de ses membres, se considérant comme appartenant à une catégorie commune ${ }^{19}$.

On peut donc poser deux conceptions de l'organisation, au gré des adaptations locales : classe ou collection. Point notable, les collections s'accompagnent de déclarations hétéro- ou auto-attributives à teneur qualifiante ou comparative.

\section{Catégorisation ou qualification : une construction ad hoc}

La vitalité idéologique du BPP se matérialise dans la langue par des formations substantivales de type prédicatif (panther inspired, panther style, panther fashion), où la fonction catégorisante est soulignée par le noyau (style, inspired). Cette dernière peut aussi prendre la forme d'emplois métaphoriques comme dans le titre de l'ouvrage Young Lords : histoire des Black Panthers latinos (1969-1976) (Richard, 2017), ou même les comparaisons initiatrices mentionnées plus haut, ou encore l'utilisation de guillemets :

"Yellow Panther" organizations formed in Vietnam and the campaigns to free Angela Davis, Huey P. Newton, and others, received national attention. (https:// digilab.libs.uga.edu/exhibits/exhibits/show/civil-rights-digital-history-p/blackbrown-power/black-nationalism, consulté le 24/04/2019)

Olivia Guérin signale :

Un nom a soit le statut de forme classifiante (un nom comme professeur indique l'appartenance du référent à une classe), soit de forme qualifiante (les noms «de qualité» ne classifient pas: Tu es un imbécile). (Guérin, 2012, p. 1086)

Mais cette autrice souligne que si certaines formes nominales sont par nature classifiantes, elles peuvent, dans certains emplois, assumer également une valeur qualifiante. C'est le cas de panther qui, associé à style, fashion, inspired, est placé dans une structure syntaxique possédant une dimension

17. Le mouvement maori amorça un tournant lorsqu'il adopta comme formule politique black, dont il faisait ainsi un élément lexical renvoyant non seulement à l'identité, mais aussi au combat contre les discriminations coloniales (voir Stastny, Orr, 2014, p. 65).

18. Selon les propres termes de J.V. Pawar, l'un des membres fondateurs des Dalit Panthers.

19. Le cas des White Panthers of the UK est plus complexe : beaucoup plus confidentiel que les autres groupes, il s'inscrit davantage dans la contre-culture que dans l'action politique de terrain. La connexion serait davantage avec le White Panther Party à travers le $\mathrm{MC}_{5}$. 
qualifiante. O. Guérin précise qu'«il ne s'agit pas uniquement d'indiquer l'appartenance à une classe, mais bien une qualité (en l'occurrence, un type d'attitude, de comportement) » (ibid.) ${ }^{20}$, le nom n'apparaissant plus en emploi référentiel, mais prédicatif21 :

Il s'agit d'une catégorisation ad hoc [...] la forme permet de bâtir une représentation spécifique du référent, en le présentant depuis un point de vue particulier. Le type de catégorisation concerné ne met pas l'accent sur la place du référent dans l'organisation du réel, mais sur des propriétés accessoires que l'observateur sélectionne, au sein d'une représentation subjective. (Guérin, 2012, p. 1092)

Le mécanisme sémantique ici mis au jour peut être rapproché de celui qui préside aux dénominations des avatars du BPP qui auraient émergé des comparaisons diversement formulées (voir supra). Elles peuvent être traitées comme des antonomases du nom propre lexicalisées et se répartiraient entre antonomase in absentia22 (Australian Black Panther Party, Polynesian Panther Party, Black Panthers of Israel) et autre forme d'antonomase référentielle plus ou moins explicite (White Panther Party, c.-à-d. White [black] Panther Party23, ou encore British Black Panther Movement). Sarah Leroy note que cette figure " construit une classe à partir des propriétés du référent originel du nom propre, et [que] ces propriétés restent les mêmes pour tous les membres de la classe» (Leroy, 2003, p.168). Est ainsi constituée une classe (ici, celle des panthers), ce qui implique une similitude plus qu'une comparaison et un fonctionnement catégorisant ad hoc ${ }^{24}$. De fait, plus qu'un parangon, l'organisation formée par B. Seale et H. P. Newton est simplement le membre initial d'une nébuleuse de mouvements d'affirmation identitaire, ainsi que l'illustre l'exemple suivant :

This Australian chapter of the Black Panther Party adapted the politics and style of the American Black Panther Party, from the clothing to their defiance, attracting the attention of the local authorities. (https://blackhistorystudies.com/resources/ resources/australian-black-panther-party/, consulté le 24/04/2019)

La forme non marquée (Black Panther Party) ne désigne pas l'organisation californienne (désignée par American Black Panther Party), mais une entité comprenant les différents avatars du BPP. Cette logique indique un lien, non

20. Perceptible dans des formations porteuses d'axiologie négative comme to pantherize, ou pantherism, marquant une certaine attitude.

21. Ce point est imputable à la possibilité qu'ont les substantifs anglais d'accéder à la fonction adnominale. Au plan sémantique, cela se traduit par «la mise en valeur de la représentation mentale du signifié en tant que matière conceptuelle, aux dépens de toute visée référentielle» (Henkel, 2014, p. 90).

22. Antonomase dont le référent-cible n'apparaît pas dans le cotexte, comme dans les exemples cités en note 10. L'antonomase in absentia est référentielle, selon S. Leroy, 2003.

23. Et non White Panther (Party), qui ouvre la voie à une interprétation suprémaciste. Comme souligné plus haut, les White Panthers of the UK rendraient davantage hommage au WPP, formant une antonomase prédicative avec ceux-ci.

24. Williams Yohuru (2006) parle de Panther mimics à propos des groupes reprenant le nom du BPP. 
pas de hiérarchie, mais d'équivalence avec le groupe de référence, et, une fois de plus, une logique de classe.

\section{Une utilisation en discours}

Les zones d'habitation de la panthère tout comme l'extension sémantique du substantif panther, signifiant aussi bien «léopard, panthère » que "puma», favorisèrent les assimilations locales en Asie $^{25}$ et en Australie ${ }^{26}$. Si l'on songe à la proposition alternative de Black Lions of Judea en lieu et place de Black Panthers of Israel, on ne peut que noter une récurrence de la métaphore féline, dont le symbolisme selon Oz Frankel (2012) dépasse l'explication initiale proposée par S. Carmichael (1966) («a beautiful black animal which symbolizes the strength and dignity of black people ») ou revendiquée par nombre de tenants du groupe (un animal qui ne recule jamais face au danger) :

Rather than the color "Black», it was the figure of the panther that facilitated the articulation of political dissent. The panther stood for masculinity, action, stealth, and aggression. (Frankel, 2012, p. 93)

Ainsi, les membres du BPP sont officiellement baptisés Panthers et Pantherettes, distinction genrée qui disparut en 1968 sous l'impulsion de militantes comme Angela Davis ou Elaine Brown, qui dénonçaient le virilisme de l'organisation. De fait, le passage au pluriel du terme panther matérialise cette identification. Ce glissement est facilité par l'adjectif de couleur; il obéit à des mécanismes similaires à ceux que subit le syntagme Brown Berets : la prégnance perceptive d'un accessoire ou d'un symbole déplaçant la référence de l'adjectif de couleur de ceux-ci à la couleur de peau.

\section{Un parti ou des panthères}

L'individualisation des membres du BPP est facilitée par ce lexème métaphorique, d'un emploi syntaxique aisé car il s'agit d'un nom commun comptable. L'ambiguïté désignative entre le parti et la somme de ses membres, "a sum corresponding to some contextually specified set of men» (Barker, 1992, p. 76), est accentuée par le fait que l'anglais autorise plus facilement que le français la syllepse grammaticale, qui fait accorder le singulier (sujet) au pluriel (verbe)

25. Ainsi, les Dalits choisirent tout autant ce terme pour la référence à l'animal et son courage qu'à l'organisation états-unienne.

26. Le mouvement maori ne put se dénommer Polynesian Black Panther Party, car une association de gangs maoris et iliens pacifiques avait déjà pris le nom de Black Panther, preuve de la prégnance de l'animal dans les représentations de la force. Il convient aussi de noter l'importance de la lutte des Africains-Américains dans les imaginaires, puisque l'un de ces gangs, vivant du trafic de drogue, se dénommait Black Power. 
ou l'inverse, comme en (1), ou encore, comme en (2), un syntagme sujet au pluriel et un attribut au singulier :

(1) The Black Panthers, also known as the Black Panther Party, was a political organization founded in 1966 by Huey Newton and Bobby Seale to challenge police brutality against the African American community. (https://www.history.com/topics/ civil-rights-movement/black-panthers, consulté le 24/04/2019)

(2) The Black Panthers rapidly grew into a national and then international party (Delli Carpini, 2000, p.193; en italique dans le texte)

Si l'on se réfère au travail de Viviane Arigne (2011), party est un nom discret collectif, ce qui signifie qu'au plan sémantique, il renvoie à un tout unitaire, ou tout intégré, caractérisé par une limite et analysable en parties qui «possèdent une forme d'autonomie, des limites non arbitraires qui sont, d'une certaine façon, motivées, ainsi qu'une fonction déterminée par rapport au tout » (Arigne, 2011, p.61).

La théorie du tout intégré considère que le nom discret collectif réunit deux types d'unités, une unité discrète de rang supérieur (ici Black Panther Party) qui englobe des sous-unités, multiples et de rang inférieur, qui sont également discrètes (ici des Panthers ou des Black Panthers). V. Arigne (2011) considère qu'en sus de ces parties homogènes entre elles (homéomères), il convient de prendre en compte le principe d'organisation, également unité de rang inférieur, mais différente et donc hétérogène par rapport à celle-ci. Elle considère que «cette hétérogénéité est ce qui fournit la limite caractéristique de ce nom et le constitue comme nom discret et comme tout intégré» (Arigne, 2011, p.63), soulignant le double statut et la saillance d'un type d'unités par rapport à une autre. Ainsi, dans :

Showdown in Desire portrays the Black Panther Party in New Orleans in 1970, a year that included a shootout with the police on Piety Street, the creation of survival programs, and the daylong standoff between the Panthers and the police in the Desire housing development. (https://www.uapress.com/product/showdown-indesire/, consulté le 24/04/2019)

la reprise anaphorique (the panthers) montre ici que la saillance tend à la pluralité. Est mis en évidence, ou le parti en tant qu'ensemble d'individus, ou ses membres en tant qu'ils s'affrontent avec la police. Mais il est d'autres cas où ce point est moins perceptible :

The Black Panther Party also built ties with leftist ethnic organizations, such as the Brown Berets (Chicano), the Young Lords (Puerto Rican), and the Red Guard (Chinese). And despite their often critical rhetoric and occasional confrontations (most seriously, with the Los Angeles-based black nationalist organization Us), the Black Panthers had reasonably good working relationships with other black organizations such as the SNCC, the League of Revolutionary Workers, and the Republic of New Africa. (Delli Carpini, 2000, p.193) 
En effet, la reprise anaphorique plurielle (acceptée par l'anglais) rend la décision complexe, d'autant qu'elle est en cataphore avec the black panthers. Their récupère le référent du nom collectif présent dans la phrase précédente dans une relation interphrastique tout en anticipant le SN au pluriel dans une relation intraphrastique (interprétation cataphorique). L'endophore 27 produit une forme de basculement interprétatif qui se fait au fil de la temporalité et de la dynamicité du discours.

Ainsi, le jeu ambigu qui mène à s'interroger sur le référent de Panther - s'agit-il des membres du parti ou du parti ? - s'explique par la difficulté à appréhender le parti en classe ou en collection.

\section{Classe ou collection?}

En discours, cette dénomination n'est en effet pas sans produire certaines équivoques, comme l'indiquent les exemples suivants :

(3) Black Panthers leader Bobby Seale was ordered to be bound and gagged (Jenner, 2016, p. 90)

(4) Black Panther leader Huey Newton holds a press conference in San Francisco after returning from a meeting with Chinese Premier Chou En-lai in China. (https:// www.viewpointmag.com/2018/06/11/intercommunalism-the-late-theorizationsof-huey-p-newton-chief-theoretician-of-the-black-panther-party/, consulté le 24/04/2019)

Comme précédemment, l'analyse permet de discriminer l'utilisation du pluriel (3), qui renverrait au leader des Black Panthers, donc de la somme des individus, de celle du singulier (4), qui renverrait à la caractérisation du personnage, en tant que membre du parti. En d'autres termes, B. Seale est aussi bien un Black Panther que le leader des Black Panthers. En effet, l'exemple Black Panthers leader Bobby Seale renvoie à la fonction de B. Seale en tant que leader des Black Panthers; il est membre des BPP, donc une collection d'individus. En revanche, Black Panther leader Huey Newton renvoie à la fonction de leader du groupe Black Panther, donc une classe. Autrement dit, dans ces exemples, H. P. Newton est un blackpanther alors que B. Seale est membre, fait partie des black panthers ${ }^{28}$. Le syntagme peut tout autant signifier une collection d'individus équivalente du parti29 qu'une classe d'individus présents dans l'espace public, avec, parfois, des formulations qui rendent cette décision interprétative peu aisée :

27. L'endophore est l'hyperonyme désignant les phénomènes anaphoriques et cataphoriques.

28. "Collections must be distinguished from classes. The class-member relation is not a meronymic relation because it is not expressed by "part" but by "is" " (Winston, Chaffin, Herrmann, 1987, p. 423).

29. "Collections whose members are determined by social connection are generally referred to as "groups"» (Winston, Chaffin, Herrmann, 1987, p.423). 
(5) The black panthers formed "police patrols", first in Oakland and then, as the organization grew, in other city around the country (Delli Carpini, 2000, p. 192)

VS

(6) The Panthers practiced militant self-defense of minority communities against the U.S. government, and fought to establish revolutionary socialism through mass (https://www.marxists.org/history/usa/workers/black-panthers/, consulté le $24 / 04 / 2019$ )

C'est globalement la cooccurrence qui permet l'interprétation en collection en raison du sème / fonction/ présent dans organization en (5). Le maintien au pluriel (ou plutôt le non-passage au singulier) conduit à une analyse en classe dans (6), la similitude catégorielle étant suggérée par la dimension distributionnelle du nombre. C'est le même type d'analyse qui conduit à analyser (7) comme une perception en collection. Cependant, (8) est moins explicite dans la mesure où la dimension inchoative du prétérit en be-ing en cooccurrence avec le verbe organizing pointe l'idée de structuration :

(7) The fall of the Panthers came just as quickly, as the organization endured the full brunt of state repression and violence (https://socialistworker.org/2016/11/03/ revolutionary-legacy-of-the-black-panthers, consulté le 24/04/2019)

(8) Panthers were organizing in Los Angeles, and that fall they stepped up their efforts to prevent police terror in black communities in the city. (Ogbar, 2006, p. 255)

On peut imputer cette ambiguïté au fait que les membres du BPP se voient attribuer, par métonymie, une dénomination (Panther). Cependant, la comparaison avec les désignations en discours de Black Beret Cadre, dont la structure est similaire et les membres dénommés selon des principes équivalents (berets), bat en brèche cette hypothèse :

Indeed, the Cadre was the true "child" of the "long sixties"; Berets had grown up in segregated Bermuda, experienced the theater boycott. (Swan, 2009, p. 95)

He supported the Berets and attended at least one Cadre meeting. (Swan, 2009, p.104)

Comme le montrent les exemples ci-dessus, les utilisations sont clairement tranchées : Cadre désigne le parti en tant que collection, alors que Berets désigne l'ensemble de ses membres en tant que classe. Il semblerait donc que si les dénominations des avatars du BPP avaient été distribuées entre classes et collections, les utilisations en discours auraient mis au jour des actualisations diverses du collectif que forme l'organisation originelle, hésitant entre l'une ou l'autre. 


\section{Une prégnance sémantique}

L'ambiguïté entre la référence aux membres du parti ou à l'organisation qu'ils constituent est donc à imputer aux réductions syntagmatiques qui apparaissent en discours, ainsi résumées:

- La forme dite pleine : the Black Panther Party

- La forme réduite à la tête : the party ou the Party (rare)

- La forme réduite à l'expansion du terme agrammatical (mais prégnant ici) : the (black) panther(s), qui dans ce cas, s'associe à un pluriel (voir supra).

Les études de statistique textuelle de Yannis Haralambous et Elisa Lavagnino (2001) ont montré que la binarité d'un terme complexe est à la base de son instabilité et des changements que sa linéarité peut subir. Ces modifications sont déterminées par les déplacements des composants ou bien leur chute, comme dans le cas de la réduction (voir Haralambous, Lavagnino, 2001, p. 4). C'est le cas de BPP qui a perdu for Self Defense, de Black Panthers qui a perdu of Israel et de Dalit Panthers qui a perdu of India.

Ce phénomène est peu étudié en dehors des travaux orientés vers la terminologie, qui le définissent comme « un mécanisme discursif qui, à travers l'élision d'au moins un constituant, transforme globalement un terme complexe en maintenant ses noyaux référentiel et notionnel» (Collet, 1997, p. 195). Ainsi que mentionné plus haut, la réduction préférentielle en discours de Black Panther Party, qu'il s'agisse de l'organisation californienne ou de ses développements hors des frontières états-uniennes est, contrairement à la norme, Panther, et non Party, ce qui ne permet pas de percevoir qu'il s'agit d'un parti politique. Ce choix peut s'expliquer logiquement, car c'est l'information non différentielle qui est supprimée (Portelance, 1996, p. 101), party ayant une amplitude trop vaste et the Party pouvant renvoyer, dans certaines aires géographiques, au Parti communiste. La suppression de black s'explique quant à elle par le fait que chutent en général les éléments qui ont cessé d'être différentiels, montrant la centralité des luttes d'affirmation comme une forme de familiarité du locuteur3o.

Ces choix préférentiels se font donc au regard de logiques d'informativité («ne pas contenir plus d'information qu'il n'est requis»), comme semblerait le corroborer l'utilisation de la réduction anaphorique Black Panthers pour représenter l'holonyme (voir supra) ${ }^{31}$. Cependant, on constate qu'en discours, the

30. En effet, ce type de réduction est prégnante chez sympathisants et proches du BPP. La centralité est la caractéristique de certains référents plus prégnants que d'autres dans une sphère d'activité donnée. Ainsi, Yellow Panther, titre du texte de Martin Wong (1998), membre asiatiqueaméricain du BPP, à interpréter comme Yellow (black) Panther. En fait, black signifie plutôt «non blanc ", ainsi que l'indique cette anecdote que rapporte Mike Tagawa, qui, expliquant qu'il ne pouvait rejoindre le BPP car il était japonais, se vit répondre : «But you ain't white either».

31. Conformément aux principes mis au jour parY. Haralambous et E. Lavagnino, la réduction anaphorique « représente l’hyperonyme du terme complexe» (Haralambous, Lavagnino, 2001, p. 44). 
(Black) Panther(s) peut également renvoyer à l'avatar local du BPP, comme le montre cette référence aux Black Panthers australiens dans le Sydney Morning Herald:

Marlene Cummins says women were abused by members of the Black Panthers (https://www.smh.com.au/entertainment/tv-and-radio/australian-black-panthersmarlene-cummins-highlights-violence-against-women-20151026-gkjod7.html, consulté le 24/04/2019)

Cet exemple, par la mise en application de la maxime de réponse sur le groupe, constitue un indicateur de l'appropriation de la dénomination par les avatars du BPP. En effet, cette maxime pose :

dès lors qu'il existe une correspondance socialement établie entre une activité et un groupe de référence, la réponse non-marquée à une question sur cette activité est la réponse qui réfère au groupe en question. (Encrevé, Fornel, 1983, p. 21)

Une entité se caractérise par ses membres et la structure qu'ils constituent. Dans le cas présent, la dialectique entre les différents niveaux (membres, organisations locales, galaxie composée des groupes se réclamant du BPP), maintenue par une forme d'ambiguïté référentielle des désignations que l'on constate au fil des discours, facilite les logiques d'adaptation locale, contribuant à la construction de cette internationale qui semble dès lors être davantage une classe d'individus (les panthers) qu'une classe d'organisations.

\section{Conclusion}

À l'issue de cet excursus, on ne peut qu'être frappé par les dialectiques entre l'hétéro- et l'auto-attribution des dénominations de la galaxie issue du BPP. L'antonomase à l'origine des dénominations des avatars du BPP californien s'appuie sur les mécanismes dialogiques à l'œuvre dans cette figure lexicale, comme le montre S. Leroy. La pratique du surnom, à la fois ironisante, catégorisante et attributive est également fonctionnelle. Liée aux logiques de contrôle social, elle constitue également un « analyseur des règles constitutives de l'interaction locale» pour reprendre une expression et réflexion de Jean-Pierre Hassoun (2000, p.34), le déni de nom constituant un acte d'une grande gravité dans les sociétés traditionnelles ou même industrielles. Le remplacement des noms par des descriptifs catégorisants, qui renvoient à des pratiques auxquelles ont été douloureusement exposées les populations africainesaméricaines trouvent un écho dans l'hétéro-dénomination séminale. La réappropriation du stigmate, pratique des groupes dominés, trouve dans cette relation de désignation passant par un mode dénominatif une forme de lien avec un groupe dont l'internationalisme est l'un des mots d'ordre. En effet, le 
double fonctionnement de Black Panther(s) en classe et en collection fonctionne non seulement pour le BPP, mais aussi pour les groupes issus de celui$\mathrm{ci}$, comme l'indique l'adjectif relationnel géographique dans l'utilisation en discours (Israeli, American, UK, etc.). La déclinaison mélanymique, ethnique, géographique ou symbolique, si l'on songe aux Gray Panthers ou les Lavander Panthers, renforce un paradigme et donne une consistance à un groupe politique. S'il n'eut de parti que le nom et les soubassements idéologiques, au sens où le terme sous-entend des mandats électifs, le BPP fut à l'origine d'une nébuleuse mondiale qui reprit plus ou moins son répertoire d'action ou ses objectifs. La dialectique entre le nom des membres du parti et le nom du parti lui-même n'est pas sans créer une certaine confusion en discours, confusion qui n'est en soi pas totalement fortuite, party étant peut-être à analyser au sens de «faction».

\section{Références}

Angelo Anne-Marie, 2009, "The Black Panthers in London, 1967-1972: A diasporic struggle navigates the Black Atlantic », Radical History Review, nº 103, p. 17-35.

ARIGNE Viviane, 2011, "La figure de tout intégré et les noms discrets collectifs », Anglophonia, no 15 (30), p. 59-99.

BARKER Chris, 1992, "Group terms in English: Representing groups as atoms », Journal of Semantics, vol. IX, nº 1 , p. 69-93.

CARMICHAEL Stockely, 1966, Black Power Address at UC Berkeley, www.americanrhetoric.com/speeches/stokelycarmichaelblackpower.html (consulté le 15 juin 2018).

CLEAVER Kathleen, KATSIAfICAS George éd., 2001, Liberation, Imagination and the Black Panther Party, Londres, New York, Routledge.

Collet Tanja, 1997, "La réduction des unités terminologiques complexes de type syntagmatique», Meta. Journal des traducteurs, vol.XLII, nº 1, p. 194-206.

Delli Carpinı Michael X., 2000, «Black Panther Party: 1966-1982», dans The Encyclopedia of Third Parties in America, I. Ness et J. Ciment éd., Armonk, Sharpe Reference, p. 190-197.

EnCREVÉ Pierre, Fornel Michel de, 1983, "Le sens en pratique», Actes de la recherche en sciences sociales, n 46, p. 3-30.

FRANKEL Oz, 2012, "The Black Panthers of Israel and the politics of the radical analogy», dans Black Power beyond Borders, N. Slate éd., New York, Palgrave Macmillan, p. 81-106.

GUÉRIN Olivia, 2012, "Catégorisation, classifiance et organisation des séquences textuelles», $3^{e}$ Congrès mondial de linguistique française, F. Neveu, V. Muni Toke, P. Blumenthal et al. éd., Les Ulis, EDP Sciences, p.1083-1097, https://www.shsconferences.org/articles/shsconf/abs/2012/01/shsconf_cmlf12_000119/shsconf_ cmlf12_000119.html (consulté le 10 mars 2019).

GUILBERT Louis, 1975, La créativité lexicale, Paris, Larousse. 
Haralambous Yannis, LaVAgnino Elisa, 2011, "La réduction des termes complexes dans les langues de spécialité», TAL, vol. LII, nº1, p. 37-68.

HASSOUN Jean-Pierre, 2000, "Le surnom et ses usages sur les marchés à la criée du Matif : contrôle social, fluidité relationnelle et représentations collectives», Genèses, n० 41, p. 5-40.

HENKEL Daniel, 2014, L'adjectif en anglais et en français : syntaxe, sémantique et traduction, thèse sous la direction de P. Cotte, Paris, Université Paris-Sorbonne.

Jenner Mareike, 2016, American TV Detective Dramas: Serial Investigations, Basingstoke, Palgrave Macmillan.

KLEIBER Georges, 2001, «Remarques sur la dénomination », Cahiers de praxématique, n०36, p. 21-41.

LEROY Sarah, 2003, «Antonomase, métaphore et nom propre : identification ou catégorisation? », Travaux linguistiques du Cerlico, n²16, p. 161-178.

LOTHIAN Kathy, 2007, «Moving blackwards: Black Power and the Aboriginal Embassy », dans Transgressions: Critical Australian Indigenous Histories, I. Macfarlane et M. Hanah éd., Canberra, ANU Press, p. 19-34.

MoIRAND Sophie, 2018, «L'apport de petits corpus à la compréhension des faits d'actualité», Corpus, n 18, http://journals.openedition.org/corpus/3519 (consulté le 9 novembre 2018).

Newton Huey P., 1968, Huey Newton Talks to the Movement about the Black Panther Party, Cultural Nationalism, SNCC, Liberals, and White Revolutionaries, Chicago, San Francisco, Students for a Democratic Society, The Movement, p. 3-14.

MURCH Donna, 2012, "When the panther travels: Race and the southern diaspora in the history of the BPP, 1964-1972 », dans Black Power beyond Borders, N. Slate éd., New York, Palgrave Macmillan, p. 57-78.

OGBAR Jeffrey O. G., 2006, «Brown power to brown people: Radical ethnic nationalism, the Black Panthers, and Latino radicalism, 1967-1973», dans In Search of the Black PantherParty, J. Lazerow et W. Yohuru éd., Durham, Duke University Press, p. 252-286.

PORTELANCE Christine, 1996, "De la nomination : catégorisation et syntagmatique», Applied Semiotics / Sémiotique appliquée, vol. l, nº 2, p. 99-108.

RiCHARD Claire, 2017, Young Lords : histoire des Black Panthers latinos (1969-1976), Paris, L'échappée.

SEALE Bobby, 1991, Seize the Time: The Story of the Black Panther Party and Huey P. Newton, Baltimore, Black Classic Press.

SHILliam Robbie, 2012, "The Polynesian Panthers and the Black Power gang: Surviving racism and colonialism in Aotearoa New Zealand », dans Black Power beyond Borders, N. Slate éd., New York, Palgrave Macmillan, p. 107-126.

Sнонат Ella, 1997, "The narrative of the nation and the discourse of modernization: The case of the Mizrahim », Critique: Critical Middle Eastern Studies, vol. VI, n ${ }^{\circ} 10$, p. 3-18.

SLATE Nico éd., 2012, Black Power beyond Borders, New York, Palgrave Macmillan.

STASTNY Angelique, ORR Raymond, 2014, "The influence of the US Black Panthers on indigenous activism in Australia and New Zealand from 1969 onwards ", Australian Aboriginal Studies, $\mathrm{n}^{\circ}$ 2, p. 60-74. 
SWAN Quito, 2009, Black Power in Bermuda: The Struggle for Decolonization, New York, Palgrave Macmillan.

UMojA Akinyele Omowale, 1999, «Repression breeds resistances: The black liberation army and the radical legacy of the Black Panther Party », New Political Science, vol.XXI, no2, p.131-155.

Winston Morton E., Chaffin Roger, Herrmann Douglas, 1987, «A taxonomy of partwhole relations », Cognitive science, vol. XI, n 4 , p. 417-444.

YoHURU Williams, 2006, "Introductory comment: White Tigers, Brown Berets, Black Panthers, Oh My! », dans In Search of the Black Panther Party, J. Lazerow et W. Yohuru éd., Durham, Duke University Press, p. 183-190.

\section{Résumé / Abstract / Compendio}

\section{Du parti de la panthère noire aux panthères : un ou des Black Panther Party(ies)?}

Cet article se propose d'exposer les variations paradigmatiques et syntagmatiques du syntagme désignatif Black Panther Party for Self Defense (1966-1982), ainsi que les enjeux politiques et discursifs de celles-ci, qu'il s'agisse du nom du parti américain ou de ses diverses adaptations locales par les divers mouvements se reconnaissant dans son discours nationaliste et marxiste. On s'aperçoit que le parti de la panthère noire devient le parti des panthères noires.

Mots-clés : affirmation identitaire, paradigme, syntagme, autodéfense, surnom

\section{From the Black Panther Party to the Panthers: one or some Black Panther Party(ies)?}

This article deals with the name Black Panther Party for Self Defense, and its variations from a discursive and political point of view. It deals with the American party, but also with different aborigenal organisations, which recognize themselves in the former's nationalist and marxist discourse. In the process, the Black Panther Party becomes the Black Panthers party.

Keywords: identity affirmation, paradigm, syntagm, self-defense, nickname

\section{Del partido de la pantera negra a las panteras: ¿uno o varios Black Panther Party(ies)?}

Este artículo tiene como propósito exponer las variaciones paradigmáticas y sintagmáticas del sintagma designativo Black Panther Party for Self Defense (1966-1982), asi como las cuestiones políticas y discursivas de aquellas, tratándose o bien del nombre del partido americano o bien de una de sus varias adaptaciones locales por los diversos movimientos que están en adecuación con su discurso nacionalista y marxista. Nos damos cuenta de que el partido de la pantera negra se convierte en el partido de las panteras negras.

Palabras claves: afirmación identitaria, paradigma, sintagma, autodefensa, apodo 